\title{
A Methodology for the Economic Assessment of Picture Archiving and Communication Systems
}

\author{
Curtis P. Langlotz, Orit Even-Shoshan, Sridhar S. Seshadri, Inna Brikman, \\ Sheel Kishore, Harold L. Kundel, and J. Sanford Schwartz
}

\begin{abstract}
Most economic studies of picture archiving and communication systems (PACS) to date, including our own, have focused on the perspectives of the radiology department and its direct costs. However, many researchers have suggested additional cost savings that may accrue to the medical center as a whole through increased operational capacity, fewer lost images, rapid simultaneous access to images, and other decreases in resource utilization. We describe here an economic analysis framework we have developed to estimate these potential additional savings. Our framework is comprised of two parallel measurement methods. The first method estimates the cost of care actually delivered through online capture of charge entries from the hospital's billing computer and from the clinical practices' billing database. Multiple regression analyses will be used to model cost of care, length of stay, and other estimates of resource utilization. The second method is the observational measurement of actual resource utilization, such as technologist time, frequency and duration of film searches, and equipment utilization rates. The costs associated with changes in resource use will be estimated using wage rates and other standard economic methods. Our working hypothesis is that after controlling for the underlying clinical and demographic differences among patients, patients imaged using a PACS will have shorter lengths of stay, shorter exam performance times, and decreased costs of care. We expect the results of our analysis to explain and resolve some of the conflicting views of the cost-effectiveness of PACS. Copyright $\odot 1995$ by W.B. Saunders Company
\end{abstract}

KEY WORDS: picture archiving and communication systems (PACS), cost effectiveness, technology assessment, intensive care unit, computed radiography.

$\mathbf{P}$ ICTURE ARCHIVING and communication systems (PACS) offer the promise of low operating costs, increased efficiency, and improved quality of care. As the technical feasibility of large-scale digital PACS becomes likely, researchers have grown interested in quantifying the costs and benefits of the new technology. Yet past experience shows that the benefits of technologic innovations in radiology may be associated with substantial additional increases in cost. ${ }^{1}$ Consequently, there is increasing interest among PACS researchers in performing economic evaluations of PACS to show their cost effectiveness.

Most economic studies of PACS to date, including our own, have not considered a variety of additional potential cost savings accruing to the medical center through increased operational capacity, rapid simultaneous access to images, and other decreases in resource use. These cost savings of PACS have not been explicitly considered in part because they do not directly affect the budget of the radiology department, and because they are difficult to measure. We are currently engaged in a large-scale study of the simultaneous implementation of PACS and computed radiography in a medical intensive care unit (MICU). We have devised a methodology to measure these previously hidden economic effects of PACS. In the sections that follow, we will outline our data collection and analysis methodology.

\section{OVERVIEW OF CLINICAL ECONOMIC ASSESSMENT METHODS}

In recent years, health-care researchers from a variety of disciplines have developed new techniques for the evaluation of the economic impact of medical technology. ${ }^{2}$ For example, economic analyses such as cost effectiveness can be used to allocate resources among widely disparate health-care strategies. ${ }^{3,4}$ These economic methods have been used to study new radiologic contrast media, ${ }^{5,6}$ a variety of imaging procedures, ${ }^{7-10}$ and many nonradiologic tests and therapies. ${ }^{11}$

Three important dimensions must be considered in the economic analysis of medical care:

From the Department of Radiology, University of Pennsylvania, and the Leonard Davis Institute of Health Economics, Philadelphia, PA.

Supported by Grant No. CA53141 from the National Cancer Institute, National Institutes of Health, United States Public Health Senice. C.P.L. is supported in part by a General Electric Radiology Research Academic Fellowship (GERRAF) Award.

Address reprint requests to Curtis $P$. Langlotz, MD, PhD, Chief, Section on Health Services Research, Department of Radiology, University of Pennsylvania, 3600 Market St, Suite 370, Philadelphia, PA 19104.

Copyright 11995 by W.B. Saunders Company

0897-1889/95/0802-0003\$3.00/0 
the perspective of the analysis (patient, payor, provider), the type of analysis (cost identification, cost benefit, or cost effectiveness), and the types of costs and benefits included (direct, indirect, and/or intangible). ${ }^{12}$ The following sections review these three perspectives.

\section{Analytic Perspectives}

Costs and benefits can be calculated with respect to many different points of view, including the patient, society, the hospital, and the hospital department. A study's perspective influences what is counted as a cost or benefit. For example, a hospital's cost of providing a service may be less than its charge. From the hospital's perspective, then, the charge is an overestimate of the resources consumed. However, if the patient must pay the full charge, that charge is an accurate reflection of the cost to the patient. Alternatively, if the hospital decreases its costs by discharging patients early, the patients' costs may increase because of the need for additional outpatient care. To minimize the effect of these cost-shifting incentives, most analyses are conducted from the societal perspective.

\section{Types of Economic Analysis}

The three most commonly used types of analysis are cost benefit, cost effectiveness, and cost identification. Cost-benefit analysis of medical care compares the costs of a medical intervention to its benefits, expressed in monetary units (eg, dollars). Cost effectiveness analysis is similar to cost-benefit analysis, except that it does not require that the cost and outcome of a medical intervention be expressed in the same units. This feature of cost effectiveness analysis is helpful in health-care analyses because health outcomes are often difficult to translate into units of currency. The results of cost effectiveness analyses generally are reported as a ratio of the number of dollars spent per unit of the relevant outcome (eg, dollars per year of life saved). A less complex approach than costbenefit or cost-effectiveness analysis is costidentification analysis, which simply enumerates the costs involved in medical care without considering the outcomes that result. By performing cost-identification analysis, the researcher can assess the costs of alternative ways of providing the same service.

\section{Types of Cost and Benefit}

Another dimension of an economic analysis considers the types of costs and benefits that it considers-direct, indirect, and intangible. The direct medical costs of care are usually associated with monetary transactions and represent costs that are incurred in providing the care. Examples of direct medical costs include payments for purchasing pharmaceutical products or radiologic equipment, salaries of health professionals, and physicians' fees. Because the charge for medical care may not accurately reflect the resources consumed, special analytic techniques may be needed to determine these direct costs. ${ }^{13}$

Indirect costs, in contrast with direct costs, do not stem from transactions for goods or services. Instead, they represent the cost of morbidity (eg, time lost from work) or mortality (eg, premature death leading to removal from the work force). They are costs because they represent the loss of opportunities to use a valuable resource, a life, in alternative ways. Intangible costs are those of pain, suffering, and grief. Because these costs are extremely difficult to measure, they are often omitted from economic analyses.

\section{PRELIMINARY ECONOMIC ANALYSES OF PACS}

According to the three dimensions of economic analysis that we discussed in the previous section, most existing economic studies of PACS can be characterized as cost-identification analyses that assess direct costs from the perspective of a radiology department. For example, several researchers have developed models of the direct monetary costs of a PACS to a radiology department. ${ }^{14}$ These analyses often compare the direct costs of PACS hardware to direct cost savings that may accrue from reductions in film supplies and support-personnel costs. Each of these studies is incremental in nature, measuring the incremental cost (or savings) associated with a change from conventional film-based image management techniques to PACS.

For example, Cywinski and Vanden Brink ${ }^{15}$ describe a cost model of PACS based on tracking studies at over 600 US institutions. Their model projects that the implementation of a commercial PACS will pay for itself in 5 years, 
and produce cost savings in subsequent years. They also suggest that a digital PACS will increase the amount of time radiologists can spend with productive patient-care activities. In a comprehensive cost model tailored to a 400 bed military health-care facility, Saarinen et al ${ }^{16}$ estimated a one-time equipment cost of $\$ 7.5$ million that would generate a positive cash flow after 9 years. Pessimistic assumptions led to positive cash flow after 17 years. In a study based on experience with a prototype digital imaging system at a Dutch hospital, ${ }^{17}$ no cost savings were projected. However, this study did not project any savings in labor costs, and estimated system acquisition costs were more than twice those suggested by other experts. ${ }^{18}$ Beard et al, ${ }^{19}$ in an analysis of several digital image management systems applied to hospitals of different sizes, found that the optimal PACS for a hospital performing 100,000 examinations per year saved nearly $\$ 1$ million per year during a 5-year payoff period.

At the University of Pennsylvania, we have performed a preliminary cost-identification analysis of PACS based on our experience with a prototype digital imaging system. ${ }^{20}$ Existing departmental expenditures were used to estimate the costs of the film-based approach. An analysis of departmental operations was conducted to estimate the equipment, supplies, and personnel needed for the operation of a PACS. We found that the implementation of a department-wide PACS would result in a modest annual cost savings when compared to the present film-based system of image management.

Our cost identification study, and those performed elsewhere, suggest that for most estimates of equipment outlays, department-wide PACS systems approximately pay for themselves in direct cost savings to the radiology department. The direct cost savings to the radiology department arise primarily from the elimination of film supply costs, including chemicals, storage space, and film-handling personnel. Thus, preliminary cost-identification studies indicate that, from the perspective of a radiology department, the direct cost savings of PACS are comparable with the costs of PACS hardware acquisition. These results may have caused institutions to hesitate committing signifi- cant resources to the implementation of untested digital imaging systems. ${ }^{21}$

\section{ADDITIONAL POTENTIAL COST SAVINGS OF PACS}

Results of an analysis from a more general perspective may identify possible cost savings that have not been considered in previous analyses. For example, a recent study suggested a $1 \%$ reduction in length of stay would avert approximately $\$ 8$ per inpatient procedure per year. ${ }^{22}$ Straub and Gur $^{23}$ used a survey of users of a radiology department to estimate the cost savings to a medical center that would result from PACS implementation. Their survey found that physicians estimated an average 15 percent of their total practice costs could be attributed to delayed access to image information. The authors used these results to estimate between $1 \%$ and $5 \%$ possible savings caused by fewer repeat examinations, decreased length of stay, and improved practice efficiency. Using these figures, they extrapolated to possible cost savings to the medical center of between $\$ 2.9$ million and $\$ 14.4$ million annually, amounts that easily offset even the most pessimistic estimates of PACS equipment cost.

As the authors suggest, these estimates should be interpreted with caution. The cost factors on which the study is based are derived from survey data, not from observational studies or from structured time-analysis questionnaires. For a hospital whose mean length of stay is 6.7 days, a mean cost-savings estimate of $3 \%$ translates to a one-day savings in length of stay for nearly one in five patients. Will this data be supported by experimental data? Are there objective data to suggest that 3 of every 100 radiologic exams are repeated for nonmedical reasons? Likewise, the potential gains in clinical practice efficiency that may accrue from PACS technology are, as yet, unknown. As the authors of the study suggest, "further studies are needed to quantify more definitively [the hidden costs of conventional] film archival and communication systems." 23

The most significant cost savings of a PACS may be its beneficial effects on the delivery of patient care. For example, a PACS may increase practice efficiency, decrease length of stay, and shorten outpatient visit times. These effects may have a substantial financial impact 
on the health-care institution. ${ }^{23,24}$ The following factors have been discussed by many authors as possible primary beneficial effects of PACS implementation ${ }^{23-27}$ : increased operational capacity, fewer lost images, and rapid simultaneous access to images.

These benefits have not been explicitly considered in most previous assessments of PACS, perhaps because they do not accrue directly to the radiology department and because they are often more difficult to estimate.

\section{AN OVERVIEW OF OUR APPROACH}

Our working hypothesis is that PACS are cost-effective replacements for conventional film-based image management systems when considered from the perspective of the medical center. To test this hypothesis, we are modeling the incremental direct costs of a PACS from the perspective of the medical center for use in a cost-effectiveness model. We are explicitly attempting to include the three factors mentioned above. Our economic analysis is part of a larger study of the effect of PACS on the clinical environment, including the effect on the quality and timeliness of care, and the effect on communications between radiologists and clinicians.

We are capturing information from a randomly selected cohort of patients in the MICU, both before and after the introduction of a PACS. When data collection is triggered for an exam, a wealth of data is obtained from a variety of sources: one-on-one interviews with medical staff, video-tape recordings of film viewing, observational studies of film and personnel flow, additional timing data from a radiology information system, and clinical information from chart review.

These data are used to perform univariate and multivariate analysis and to build cost models. These models can be integrated with models of the incremental medical effectiveness of PACS implementation. Figure 1 illustrates schematically the expected form of the conclusions of the cost-effectiveness study. The $\mathrm{X}$-axis represents cost, and the $\mathrm{Y}$-axis signifies diagnostic value or effectiveness. The two points represent the best estimates of dollar cost and healthcare benefit of film-based imaging and PACS. Note that each point is surrounded by a confidence region that indicates the uncertainty in the results.

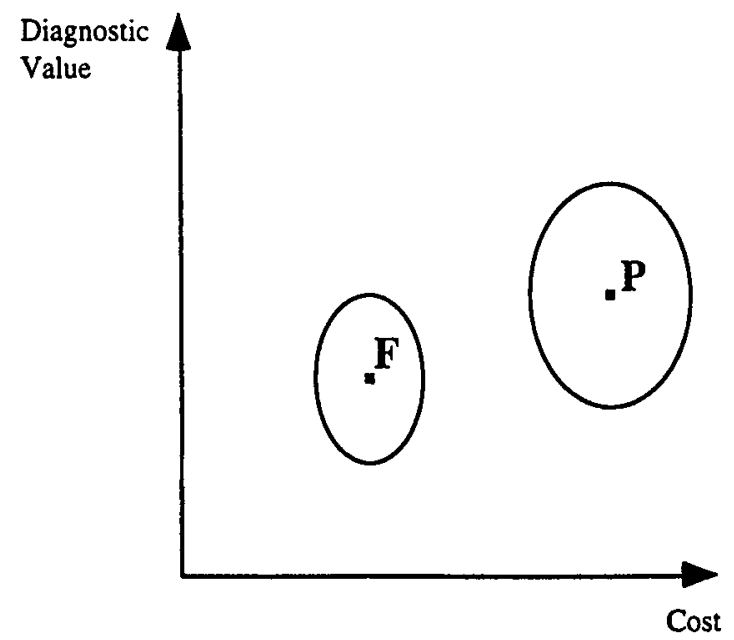

Fig 1. The overall conclusions of a study of the incremental cost effectiveness of PACS from the perspective of a medical center. The $X$-axis signifies cost; the $Y$-axis represents diagnostic value or effectiveness. Point $F$ denotes film-based image management, and point $P$ represents PACS. Ellipses illustrate confidence regions.

The economic methods we describe here are focused on locating the position of film-based and PACS-based operation along the $\mathrm{X}$-axis. Our analysis includes a measurement of the effect of PACS on (1) the capital and operating costs of a radiology department; (2) the work efficiency of clinicians, support personnel, and radiologists; (3) the length of stay of MICU patients; and (4) the costs of care delivered, including the costs of radiologic examinations, physician consultations, and the use of ancillary services.

Subsequent sensitivity analysis will assess the robustness of the results and suggest those variables that warrant additional study. In the sections that follow, we describe each component of our methodology.

\section{EXTENDING THE ECONOMIC ANALYSIS \\ TO THE MEDICAL CENTER PERSPECTIVE}

\section{Radiology Cost Identification}

The first component of our analysis is a revision of the type of cost identification study that we and others have performed in the past. This analysis will determine the effect of PACS implementation on the direct costs of our radiology department. Capital, operating, and overhead costs will be accounted for separately. Hardware and software costs will be measured in base-year dollars and depreciated over their 
useful life. The true cost of the hardware and software that has been developed in collaboration with the hospital will be estimated using the University's indirect cost rate. Actual departmental expenditures will be obtained to estimate the costs of each approach. To estimate PACS costs, the need for equipment, supplies, and personnel will be assessed. PACS equipment costs will include interpretation/review displays, image-acquisition devices, imagecompression devices, archive systems, opticaldisk platters, and film-hardcopy systems. These costs will be allocated at volume. That is, the cost will be computed per imaging examination. The rapidly declining cost of this equipment will be considered explicitly.

We are using two additional methods to extend this initial analysis to the incremental cost (or incremental savings) of PACS from the perspective of the medical center. One is to measure the cost of care actually delivered through the capture of billing entries for each patient in our study cohort. Any effects of PACS on the care actually delivered, such as decreased length of stay, decreased adverse events, or decreased utilization of radiology services, will affect the amount of care delivered, and thereby its cost. But this measure of cost will not reflect efficiencies in the provision of each unit of care (for example, less technologist time needed per portable chest examination). Consequently our second method to estimate the incremental cost of PACS is to measure changes in resource utilization in the MICU and the radiology department.

\section{Cost Estimation Through Capture of Billing Entries}

We have arranged for electronic transfer of all billing entries from the hospital billing computer and from the clinical practice billing database to our own relational research database. These entries reflect the amount of care (ie, the number of units of care) actually delivered to each patient in our study. Our database contains all charge entries for the patients in question. We also collect ancillary clinical and demographic data (such as age, sex, and admission diagnosis) and compute case mix adjusters (such as the APACHE III score ${ }^{28}$ and the Charlson score ${ }^{29,30}$ ).

Billing entries reflect not costs, but charges- unreliable estimates of the true cost of care..$^{13}$ Consequently, we are experimenting with several different approaches to convert each charge entry to its corresponding cost: an existing cost accounting system can estimate resource usage associated with each billing entry; Medicare ratios of costs to charges can provide a rough conversion between costs and charges; allowed charges from large insurers, such as Medicare or Blue Cross/Blue Shield, can be used as a proxy costs.

After making these conversions, the total cost of care delivered is estimated simply by multiplying the units of care delivered by the cost per unit. Analysis of the resulting cost data will include both univariate and multivariate techniques. Univariate analyses will be performed on the demographic data, cost of care, length of stay, exam performance time, interval between exam completion and clinical action, and filmloss rate.

Multivariate techniques offer a special advantage in a study with the number of covariates likely in this design because a multivariate model can help to discriminate the degree to which PACS may have affected the cost of care, independent of the case-mix variables that we know have an effect on the cost of care (ie, illness severity, comorbidities, and sociodemographics). To achieve these results, multivariate regression models will be constructed with cost as a dependent variable. Independent variables will include not only PACS versus film, but also selected case-mix adjusters. These incremental direct-cost models will measure the effect of PACS on medical-center costs while controlling for case mix. Similar multivariate models will be constructed to model the independent effect of PACS on length of stay. These models will test whether, after controlling for the underlying clinical and demographic differences among patients, patients imaged during the PACS periods have shorter lengths of stay, shorter exam performance times, and decreased costs of care.

\section{Cost Estimation Through Measurement of Resource Utilization}

Some changes in resource utilization, such as savings in technologist time per exam or savings in clinician search time per exam may not be reflected by billing entries. However, these 
savings do reduce costs (and may ultimately affect future decisions about the charge for an examination.)

The "life of a film" is a useful framework within which to consider these tasks. In our hospital, a portable chest examination is requested by the clinician on the hospital unit (in this case, the MICU). Next, the unit's clerical staff transmit the order to the radiology department. The technologist receives the order, travels to the patient's bedside to perform the exam, then returns with the image cassettes. After the films are created, the film images are brought to the radiologist for interpretation. The clinician later searches for the images and may confer with the radiologist. Finally, the clinician makes a decision for action (or inaction) based on the results of the examination.

We use a variety of data sources to collect information about resource utilization at each step in this process, including radiology information system (RIS) records, physician interviews, video cameras, chart reviews, and observational studies.

The RIS records, via barcode reader, the time at which radiology personnel execute many operational functions, including completing an examination and hanging the films. In addition, we track the following data from the RIS database: the number of exams repeated because of poor image quality or lost exams, the number of delayed final reports, and the number of examinations completed but never billed for.

Physician interviews are the mainstay of data collection for our overall study of PACS. We depend on this interview for estimates of (1) physician film-search time (eg, number of trips to the radiology department, time spent search- ing for films), (2) time of first image encounter, (3) frequency of lost films, and (4) actions taken as a result of the exam.

Video cameras are installed to monitor the reading boards on which MICU exams are displayed. These videotapes confirm the time and number of clinician film-search trips and the frequency of conferences between the radiologist and clinician.

Chart review is used at the bedside to document the time that actions were taken as a result of films, and to compute the APACHE III score on admission to the unit. Charts are reviewed again at the time of discharge to discover adverse events, follow short-term outcome, and identify additional case-mix variables.

Observational studies are expensive, and represent a last resort for us. However, we have used brief observational studies to assess critical variables that cannot be measured in other ways. For example, we have used these studies to document the frequency of lost films, to measure film-search times, and to clock each of the technologist's subtasks associated with performing an examination.

Table 1 summarizes how these data-collection methods are used to measure changes in personnel time, equipment utilization, and supply costs associated with each step in the life of a film.

\section{Relationship Between Charge Entries and Resource Utilization}

The two measures of cost we selected (billingentry capture and resource utilization measurement) are complementary tools for cost modeling. For example, the overall decrease in time between exam ordering and action decisions (eg, to reposition a replaced endotracheal tube),

Table 1. Data Sources for the Economic Assessment of PACS

\begin{tabular}{|c|c|c|c|c|c|c|}
\hline Exam Task & Personnel Needed & RIS & Physician Interviews & Videotape Monitoring & Observational Studies & Chart Review \\
\hline Create order & Clinician & & $x$ & & & $x$ \\
\hline Transmit order & Clerk & $x$ & & & & \\
\hline Perform exam & Technologist & $x$ & & & $x$ & \\
\hline Process film & Technologist or aide & $x$ & & & $x$ & \\
\hline Interpret exam & Radiologist & $x$ & & & & \\
\hline Search for exam & Clinician or Radiologist & & $x$ & $x$ & $x$ & \\
\hline Confer & Clinician and Radiologist & & $x$ & $x$ & & \\
\hline Action & Clinician & & $x$ & & & $x$ \\
\hline
\end{tabular}

Row labels indicate each step in the life of a film and the personnel involved in that step. Column labels indicate the data collection methods used to identify changes in resource utilization during each step. 
may result in fewer adverse events, and better patient outcomes, thereby decreasing the units of care that need to be delivered to each patient (reflected in fewer billing entries).

On the other hand, some changes in cost would not be reflected immediately by billing entries. For example, if it takes less technologist time to process and produce each exam, fewer technologists would be needed per exam, thus saving wage costs for the medical center, and decreasing its cost per exam. Ultimately, the medical center might decrease the charge for that examination. A similar argument could be made if film-search time is decreased. Thus, both of these methods are needed to capture all the costs and savings of PACS.

\section{Integrating Economic Models and Effectiveness Estimates}

In a separate analysis that is part of our overall study of PACS, (not discussed here) the diagnostic value and the utilities of PACS-based imaging will be determined. We plan to develop a cost-effectiveness ratio whereby the incremental cost of PACS (relative to film-based imaging) is the numerator, and the additional diagnostic value of PACS (relative to film-based imaging) is the denominator. This ratio will represent the incremental dollar cost per unit of added information. We recognize that some observers prefer more explicit, concrete measures of outcome. Therefore, we also will combine cost measurements with the equivalent measures of health outcome. This calculation will provide a measure that can be compared to other research on the cost-effectiveness of medical care interventions (eg, the cost per qualityadjusted life-year offered by a pharmaceutical therapy).

\section{Interpreting the Results of the Cost-Effectiveness Model}

We plan to conduct sensitivity analysis on our cost models to test their robustness. During sensitivity analysis, some of a model's parameters are varied within a reasonable range, and the results of the model are repeatedly recalculated to determine the effect of uncertainty in a model parameter on the overall results of the model. By showing the independence or dependence of a result on a particular assumption, by establishing the minimum or maximum value of a parameter that would affect the decision to adopt a technology, or by identifying uncertainties that require additional investigation, sensitivity analysis may provide evidence for or against the generalizability of our study to other health-care facilities that attract a different patient mix or that require a different PACS configuration.

\section{CONCLUSION}

We have described a dual approach to the estimation of the economic ramifications of PACS on a medical service. Our approach is cognizant of the distinction between costs and charges. We are measuring changes in billing entries, length of stay, adverse events, and utilization of radiology services. In addition, we are measuring change in the utilization of selected resources that affect the cost of each unit of care that are unlikely to be reflected in billing entries. We believe that these two methods will allow a shift in perspective from the radiology department to the medical center that will show additional savings of PACS. We expect the interpretation of our results to explain and resolve some of the conflicting views of the cost effectiveness of PACS.

\section{REFERENCES}

1. Evens RG: The economic impact of technology on diagnostic imaging at a university medical center. Am J Radiol 153:179-183, 1989

2. Eisenberg JM: Clinical economics: A guide to the economic analysis of clinical practices. JAMA 262:28792886,1989

3. Drummond MF, Stoddart GL, Torrance GW: Methods for the Economic Evaluation of Health Programmes. Oxford, UK, Oxford University, 1987

4. Eisenberg JM, Koffer H, Finkler SA: Economic analysis of a new drug: Potential savings in hospital operating costs from the use of a once daily regimen of a parenteral cephalosporin. Rev Infect Dis 6:S909-S923, 1984 (suppl, abstr)

5. Goel V, Deber RB, Detsky AS: Nonionic contrast media: Economic analysis and health policy development. Can Med Assoc J 140:389-395, 1989

6. Fischer HW: Cost vs. safety: The use of low-osmolar contrast media. JAMA 260:1614, 1986

7. Mooney G: Breast cancer screening: A study in costeffectiveness analysis. Soc Sci Med 16:1277-1283, 1982

8. Simon DG, Lubin MF: Cost-effectiveness of computerized tomography and magnetic resonance imaging in dementia. Med Decis Making 5:335-354, 1985 
9. Forman HP, Heiken JP, Brink JA, et al: CT screening for comorbid disease in patients with prostatic carcinoma: Is it cost effective. Am J Radiol 162:1125-1128, 1994

10. Yin D, Baum RA, Carpenter JP, Langlotz CP, et al: Cost effectiveness of magnetic resonance angiography in symptomatic peripheral vascular disease. Radiology 1995 (in press)

11. Detsky AS, Naglie GI: A clinician's guide to cost effectiveness analysis. Ann Intern Med 113:147-154, 1990

12. Bombardier C, Eisenberg JM: Looking into the crystal ball: Can we estimate the lifetime cost of rheumatoid arthritis? J Rheum 12:201-204, 1985

13. Finkler SA: The distinction between costs and charges. Ann Intern Med 96:102-109, 1982

14. Becker SH, Arenson RL: Costs and benefits of picture archiving and communication systems. J Am Med Informatics Assoc 1:361-371, 1994

15. Cywinski J, Vanden Brink JA: Review of experience with a PACS cost analysis model. SPIE Medical Imaging III: PACS System Design and Evaluation, Newport Beach, CA, January 29-February 3, 1989

16. Saarinen AO, Haynor J, Loop J: Modeling the economics of PACS: What is important? SPIE Medical Imaging III: PACS System Design and Evaluation, Newport Beach, CA, January 29-February 3, 1989

17. Andriessen JHT, ter Haar Romeny BM, Binkhuysen FH: Savings and costs of a picture archiving and communication system in the University Hospital Utrecht. SPIE Medical Imaging III: PACS System Design and Evaluation, Newport Beach, CA, January 29-February 3, 1989

18. van Gennip E, Ottes FP, Poppel BM: Why do cost-benefit studies of PACS disagree? SPIE Medical Imaging III: PACS System Design and Evaluation, Newport Beach, CA, January 29-February 3, 1989

19. Beard DV, Parrish D, Stevenson D: A cost analysis of film management and four PACS systems based on Ethernet, FDDI, BISDN, and HPPI. SPIE Medical Imaging III: PACS System Design and Evaluation, Newport Beach, CA, 1990
20. Seshadri SB, Arenson RL, DeSimone D: Costsavings associated with a digital radiology department: A preliminary study. Ninth Conference on Computer Applications in Radiology, Hilton Head, SC, 1988

21. Vanden Brink J, Cywinski J, Szerlag C: Cost analysis of present methods of image management. SPIE Medical Imaging III: PACS System Design and Evaluation, Newport Beach, CA, February 5, 1987

22. Stockburger W, King W: PACS: A financial analysis for economic viability. Appl Radiol 19:17-24, 1990

23. Straub WH, Gur D: The hidden costs of delayed access to diagnostic imaging information: Impact on PACS implementation. Am J Radiol 155:613-616, 1990

24. Hilsenrath PE, Smith WL, Berbaum KS: Analysis of the cost effectiveness of PACS. Am J Radiol 156:177-180, 1991

25. De Simone D, Kundel HL, Arenson RL: Effect of a digital imaging network on physician behavior in an intensive care unit. Radiology 169:41-44, 1988

26. Parrish D, Beard DV, Kilpatrick KE: Operational modeling for PACS: How do we decide if it's cost effective? SPIE Medical Imaging III: PACS System Design and Evaluation, Newport Beach, CA, January 29-February 3, 1989

27. Taira RK, Mankovich NJ, Boechat MI, Kangarloo H, Huang HK: Design and implementation of a picture archiving and communication system for pediatric radiology. Am J Radiol 150:1117-1121, 1988

28. Knaus WA, Wagner DP, Draper EA, et al: The APACHE III prognostic system: Risk prediction of hospital mortality for critically ill hospitalized adults. Chest 100:16191636, 1991

29. Romano PS, Roos LL, Jollis JG: Adapting a clinical comorbidity index for use with ICD9-CM administrative data: Differing perspectives. J Clin Epidemiol 46:1075-1079, 1993

30. Charlson ME, Pompei P, Ales KL, et al: A new method of classifying prognostic comorbidity in longitudinal studies: Development and validation. J Chron Dis 40:373383,1987 